\title{
miR-124 suppresses the migration and invasion of glioma cells in vitro via Capn4
}

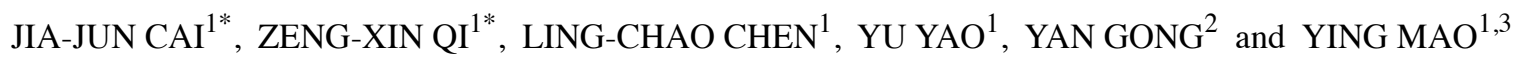 \\ Departments of ${ }^{1}$ Neurosurgery and ${ }^{2}$ Geriatrics, Huashan Hospital, Fudan University, \\ Shanghai 200040; ${ }^{3}$ Institutes of Biomedical Sciences, Fudan University, \\ State Key Laboratory of Medical Neurobiology, Shanghai 200032, P.R. China
}

Received June 3, 2015; Accepted July 13, 2015

DOI: $10.3892 /$ or.2015.4355

\begin{abstract}
R-124 and Capn4 are aberrantly expressed in glioblastoma multiforme (GBM) tissues. In the present study, we investigated miR-124 and Capn4 expression in GBM tissue specimens. The role of miR-124 and Capn 4 in the migration and invasion of glioma cells in vitro was also examined. miR-124 and Capn 4 expression in 20 GBM and 6 control brain specimens was examined using RT-qPCR and immunoblotting. Data from The Cancer Genome Atlas were retrieved. Candidate mRNA target sites of miR-124 were predicted using TargetScan/microRNA and binding was examined using dual luciferase reporter assays. The U87 and U251 cells were transfected with scrambled microRNA, miR-124 mimics and/or pLenti-Capn 4 prior to wound-healing and Transwell invasion assays. Proteins involved in the epithelial-mesenchymal transition were examined using immunoblotting. The results showed that miR-124 was significantly downregulated in GBM tissues. Immunoblotting showed a marked upregulation of Capn4 expression in GBM tissues. The Spearman's correlation analysis revealed a negative association between miR-124 expression and Capn 4 protein levels. TargetScan/microRNA predicted the miR-124 binding site in the nucleotide $440-446$
\end{abstract}

Correspondence to: Dr Yu Yao, Department of Neurosurgery, Huashan Hospital, Fudan University, 12 Wulumuqi Middle Road, Shanghai 200040, P.R. China

E-mail: yu_yao03@126.com

Dr Yan Gong, Department of Geriatrics, Huashan Hospital, Fudan University, 12 Wulumuqi Middle Road, Shanghai 200040, P.R. China

E-mail: gongyan8811@sina.com

${ }^{*}$ Contributed equally

Abbreviations: GBM, glioblastoma multiforme; miRNA, microRNA; 3'-UTR, 3' untranslated region; TGCA, The Cancer Genome Atlas

Key words: glioblastoma, Capn4, miR-124, invasion, mesenchymal phenotype region within the Capn4 3'-UTR, which was confirmed by luciferase assays. Wound-healing and Transwell invasion assays demonstrated that Capn4 downregulation or miR-124 mimics suppressed the migration and invasion of glioma cells. Capn4 downregulation or miR-124 mimics reduced the level of phospho-FAK and MMP2, vimentin and N-cadherin in U87 cells. In conclusion, miR-124 was found to suppress the migration and invasion of glioma cells in vitro via Capn4.

\section{Introduction}

Glioblastoma multiforme (GBM) is one of the most malignant and difficult-to-treat tumors. Despite the availability of multi-modality treatment involving surgical resection followed by adjuvant radio- and chemotherapy, the outcome of GBM patients remains poor with a median survival of $\sim 15$ months $(1,2)$. GBM patients succumb to brain tumor due to rapid, aggressive, and infiltrative growth of the tumor, rendering the tumor unresectable at the time of diagnosis in many cases (3).

MicroRNAs (miRNAs), a class of endogenous small non-coding RNAs of $\sim 23$ nucleotides, play key roles in regulating various biological processes $(4,5)$, and are involved in glioma migration and invasion (6-8). miR-124 is abundantly expressed in normal brain tissue and plays a crucial role in the differentiation and function of neurons, and contributes to the pathogenesis of human brain disorders (9). Several studies indicated a correlation between the downregulation of miR-124 and tumor grading in GBM (6-8). These studies revealed a lower miR-124 expression in GBM with higher invasiveness (10).

The calpains are a family of calcium-dependent neutral cysteine proteases and are active participants in processes such as cell mobility and cell cycle progression. Calpain small subunit 1 (Capn4) plays an essential role in maintaining calpain stability and activity (11). Capn4 overexpression was observed in intrahepatic cholangiocarcinoma tissues and involved in the metastasis of intrahepatic cholangiocarcinoma (12). Higher Capn4 expression was also identified in and associated with poor overall survival (OS) of clear cell renal cell carcinoma patients (13). In a previous study, we also observed an increased expression of Capn4 in glioma tissues, which was associated with a poorer OS and progression-free survival (PFS) of GBM patients. Additionally, the downregulation of 
Capn4 suppressed the invasion and migration of glioma cells in vitro (14). A tissue microarray study by Bai et al revealed a correlation between Capn4 expression and an invasive phenotype of hepatocellular carcinoma (15).

Although miR-124 and Capn4 are invovled in the invasiveness of GBM and other tumors, a connection between miR-124 and Capn 4 has not been previously documented. In the present study, we investigated the expression of miR-124 and Capn 4 in 20 GBM tissue specimens and 6 control tissue specimens and in queried data on glioma from The Cancer Genome Atlas (TCGA). It was found that miR-124 was downregulated whereas Capn4 was upregulatedin GBM tissues. miR-124 expression correlated negatively with Capn4 protein levels. Our computer algorithm analysis revealed that Capn 4 is a candidate target gene of miR-124. The results also indicated that miR-124 suppressed the migration and invasion of glioma cells in vitro by modulating Capn4.

\section{Materials and methods}

Tissue specimen acquisition. Twenty archived GBM specimens were obtained from the tissue bank of Huashan Hospital, Fudan University, Shanghai, China. These specimens were obtained from GBM patients who underwent radical surgical resection between January 2011 and December 2012 at the Department of Neurosurgery, Huashan Hospital, and snap-frozen. Six control brain specimens were obtained from trauma patients or patients who received epilepsy surgery in the interim. Acquisition of tissue specimens was approved by the hospital Institutional Review Board and was performed in accordance with the institutional and national regulations.

TCGA data retrieval. To investigate the expression of miR-124 and Capn 4 in human GBM and normal brain tissues, we retrieved glioma data on TCGA (https://tcga-data.nci.nih. gov/tcga/). The results were based on data generated by the TCGA Research Network: http://cancergenome.nih.gov/. The resulting data set was filtered for samples having expression data for miR-124 and Capn4.

Computational algorithm analysis. The in silico prediction of predicted mRNA target sites of miR124 on human mRNA transcripts was performed using the algorithms TargetScan Human 5.1 (www.targetscan.org) and human microRNA (http://www.microrna.org/). The minimum free energy predicted for hybridization was detected using BibiServ (http://bibiserv.techfak.uni-bielefeld.de/genefisher2/).

Cells and transfection. HEK293T and GBM cells, U251 and U87 (Cell Bank of Chinese Academy of Sciences, Shanghai, China), were cultured in DMEM with $10 \%$ fetal bovine serum (Gibco, Carlsbad, CA, USA).

Capn4 was knocked down by shRNA in the U251 and U87 cells as previously described (14). The cells were transfected with plasmid vectors expressing Capn4, pLenti-Capn4 or miR-124 mimics (Genomeditech, Shanghai, China) using Lipofectamine 2000 (Invitrogen, Carlsbad, CA, USA).

Dual luciferase reporter assays. The 3'-untranslated region (3'-UTR) of Capn 4 containing the miR-124 target sequence
(5'-GTGCCTT-3') was synthesized (Genomeditech) and inserted into the $X b a$ I site of the pGL3-control vector (Promega, Madison, WI, USA), with the resultant plasmids pGL3-Capn4-wt. To determine sequence specificity, the plasmid pGL3-Capn4-mt was constructed, in which the conserved targeting sequence of miR-124 was mutated to 5'-ACACTCC-3'. miR-124 mimics or scrambled miRNA and reporter plasmids were co-transfected into HEK-293 cells using Lipofectamine 2000. After 48 h, the cells were assayed with a dual-luciferase reporter assay system (Promega). Firefly luciferase activity for each sample was normalized to Renilla luciferase activity. Transfection was repeated three times in triplicate independently.

Reverse transcription-quantitative PCR. Total RNA isolation, cDNA synthesis and reverse transcription-quantitative PCR (RT-qPCR) was performed as previously described (14), using the primers: Capn4, 5'-ACCCACTCCGTAACCTC-3' (sense) and 5'-GGGTAGCAACCGTGAA-3' (antisense), GAPDH, 5'-TCCACCACCCTGTTGCTGTA-3' (sense) and 5'-ACCACA GTCCATGCCATCAC-3' (antisense), for miR-124 (DHM0032, Takara, Dalian, China) and the universal miRNA control primer (D354, Takara). PCR was performed in triplicate using the ABI PRISM 7900 Sequence Detection System (Applied Biosystems, CA, USA). The relative amount of Capn 4 mRNA, standardized by GAPDH mRNA, was expressed as $-\triangle \mathrm{CT}=[\mathrm{CT}$ (Capn4)-CT (GAPDH)]. The ratio of the number of Capn4 mRNA copies to the number of GAPDH mRNA copies was then calculated as $2^{-\Delta \mathrm{CT}} \mathrm{XK}$, where $\mathrm{K}$ is a constant.

Western blot assays. Western blotting was performed as previously described (14) using the antibodies: Capn4 (Abcam, MA, USA), E-cadherin, $N$-cadherin, vimentin, MMP2, MMP9, FAK, and phospho-FAK (all from Cell Signaling Technology, Beverly, MA, USA) and $\beta$-actin (Abcam). The protein expression was normalized against $\beta$-actin and densitometric analysis was performed using Image Pro plus 6.0.

Wound-healing and Transwell invasion assays. Wound-healing and Transwell invasion assays were performed as previously described (14). Briefly, the cells were seeded in 24-well plates and when the cells were $80-90 \%$ confluent, the monolayer was scratched manually with a plastic pipette tip across the diameter of each plate. After two washes with PBS, the cell migration was observed by microscopy at $48 \mathrm{~h}$. Transwell cell invasion assays were performed using 24-well Transwells (8- $\mu$ m pore size; Millipore, Billerica, MA, USA) precoated with Matrigel (BD Biosciences, San Jose, CA, USA). A total of $2 \times 10^{5}$ cells were suspended in $200 \mu \mathrm{l}$ DMEM containing $1 \%$ FBS and added to the upper chamber. The lower chamber was filled with $600 \mu \mathrm{l}$ DMEM containing $10 \%$ FBS acting as chemoattractants. After $24 \mathrm{~h}$ of incubation, Matrigel and the cells remaining in the upper chamber were removed by cotton swabs. The cells on the lower surface of the membrane were fixed in $4 \%$ paraformaldehyde and stained with Giemsa. Cells in 5 microscopic fields were counted and imaged and expressed as the average number of cells per field of view. The experiments were performed in triplicate at least three times independently.

Statistical analysis. Data are presented as median and range or mean \pm SD where appropriate and analyzed using Student's 
A

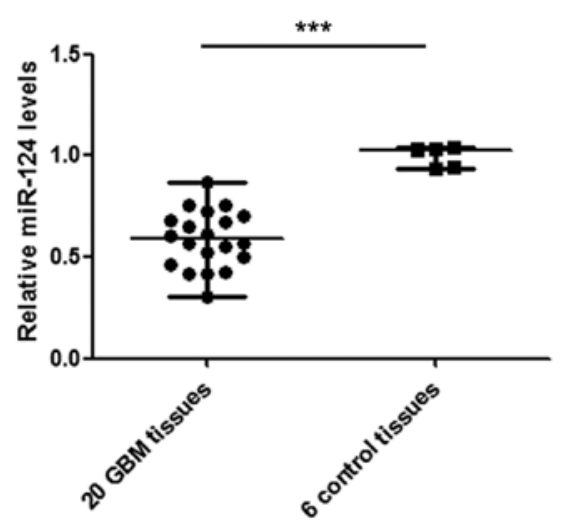

B

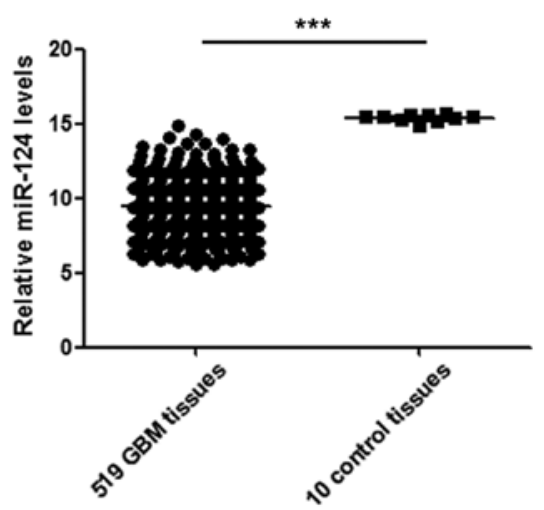

Figure 1. miR-124 is significantly downregulated in glioblastoma multiforme (GBM) tissues. (A) RT-qPCR assays were performed to determine miR-124 levels in 20 GBM and 6 normal brain tissue specimens. (B) Data from the TCGA were investigated, yielding 519 GBM and 10 normal unmatched brain tissue specimens. Data are shown as median and range. ${ }^{* * *} \mathrm{P}<0.001, \mathrm{GBM}$ vs. controls.

t-test for comparisons between groups. Spearman's correlation analysis was performed between the Capn4 mRNA level, Capn4 protein level and miR-124 level. $\mathrm{P}<0.05$ was considered statistically significant.

\section{Results}

miR-124 is significantly downregulated in GBM tissues. Analysis of $20 \mathrm{GBM}$ tissue and 6 control tissue specimens by RT-qPCR showed a median of 0.59 (range, 0.31-0.87) for GBM and 1.03 (range, 0.94-1.04) for control brain tissues $(\mathrm{P}<0.001)$ (Fig. 1A). The queried data revealed a markedly lower level of miR-124 in GBM tissues $(n=519)$ than that of normal controls $(\mathrm{n}=10)(\mathrm{P}<0.001)$ (Fig. 1B).

miR-124 directly targets the 3'-UTR of Capn4. To determine the role of miR-124 in GBM. We searched for candidate target genes of miR-124 using TargetScan and human microRNA database. We found that the nucleotide 440-446 region within the 3'-UTR of the Capn4 gene contained a candidate binding site for miR-124 (Fig. 2A). The BibiServ analysis revealed that the free energy (DG) was approximately $-17.0 \mathrm{kcal} / \mathrm{mol}$ for hybridization of the Capn4 3'-UTR and miR-124 (Fig. 2B). The homology analysis additionally revealed that the nucleotide 440-446 region within the Capn4 3'-UTR was highly conserved across species (Fig. 2C). The luciferase assays showed that HEK293T cells co-transfected with pGL3-Capn4wt and miR-124 mimics expressed significantly decreased luciferase activities compared to the cells co-transfected with pGL3-Capn4-mt and miR-124 mimics or pGL3-Capn4-wt and scrambled miRNA controls (Fig. 2D). These results demonstrated that miR-124 directly targeted the 3'-UTR of Capn4.

Capn4 is upregulated in GBM tissues at the post-transcriptional level. Given that the dysregulated expression of Capn4 has been shown to be a predictor of adverse prognosis of glioma patients (14) and our finding that Capn4 was a direct target of miR-124, we examined whether Capn4 was dysregulated in GBM. The RT-qPCR analysis demonstrated a median Capn4 mRNA transcript level of 1.06 (range, 0.99-1.44) for 20 GBM tissue specimens and 1.03 (range, 0.91-1.12) for 6 control brain tissue specimens ( $\mathrm{P}>0.05)$ (Fig. 3A). Query of data from the TCGA revealed a median Capn 4 mRNA transcript level of 10.21 (range, 6.73-11.48) for 519 GBM tissue specimens and 10.25 (range, 10.04-10.52) for 10 normal brain tissue specimens ( $\mathrm{P}>0.05)$ (Fig. 3B). By contrast, the immunoblotting assays showed a median Capn4 level of 1.60 (range, 0.32-2.22) for GBM tissue specimens and 0.26 (range, 0.01-0.40) for normal specimens $(\mathrm{P}<0.001)$ (Fig. 3C). Spearman's correlation analysis found no significant correlation between the mRNA transcript levels and protein levels of Capn4 in GBM tissues $(r=0.2227, \mathrm{P}>0.05)$ (Fig. 3D).

In addition, we transfected U87 and U251 cells with miR-124 mimics or scrambled miRNA controls (Fig. 3E). The RT-qPCR analysis revealed that miR-124 failed to markedly suppress the endogenous expression of Capn 4 mRNA transcripts compared to the cells transfected with scrambled miRNA controls ( $\mathrm{P}>0.05)$ (Fig. 3F). Consistent with our findings in GBM tissues, miR-124 mimics significantly reduced the expression of Capn4 protein in the U87 and U251 cells ( $\mathrm{P}<0.001$ vs. scrambled miRNA controls) (Fig. 3G). The above findings together indicated that Capn 4 was upregulated in GBM cells or tissues at the post-transcriptional level and not at the transcriptional level.

miR-124 expression correlates negatively with Capn4 protein levels in GBM. We examined whether miR-124 expression correlated with Capn4 expression in GBM tissues. The Spearman's correlation analysis revealed that Capn 4 mRNA transcript levels did not correlate with miR-124 levels in GBM tissues from our cohort ( $\mathrm{r}=0.2724, \mathrm{P}>0.05)$ (Fig. 4A). Analysis of the queried data from the TCGA also failed to reveal significant correlation between Capn4 mRNA transcript levels and miR-124 levels in GBM tissues ( $r=0.0134, \mathrm{P}>0.05)$ (Fig. 4B). By contrast, a significant negative correlation was demonstrated between Capn4 protein levels and mR-124 levels in the 20 GBM tissue specimens from the present study $(r=0.5352$, $\mathrm{P}<0.05$ ) (Fig. 4C).

miR-124 suppresses the migration and invasion of glioma cells by modulating Capn4. miR-124 has been shown to inhibit the migration and invasion of U87 and U251 cells (7). 
A http://www.targetscan.org/

\begin{tabular}{|c|c|}
\hline Position 440-446 of CAPNS1 3' UTR & 5' ...GACCCAGGUGCCCCAGUGCCUUU.... \\
\hline hsa-m & CCGUAAGUGGCGCACGGAAU \\
\hline
\end{tabular}

http://www.microrna.org/
B

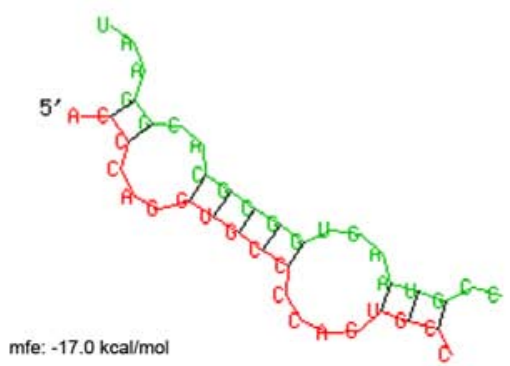

C

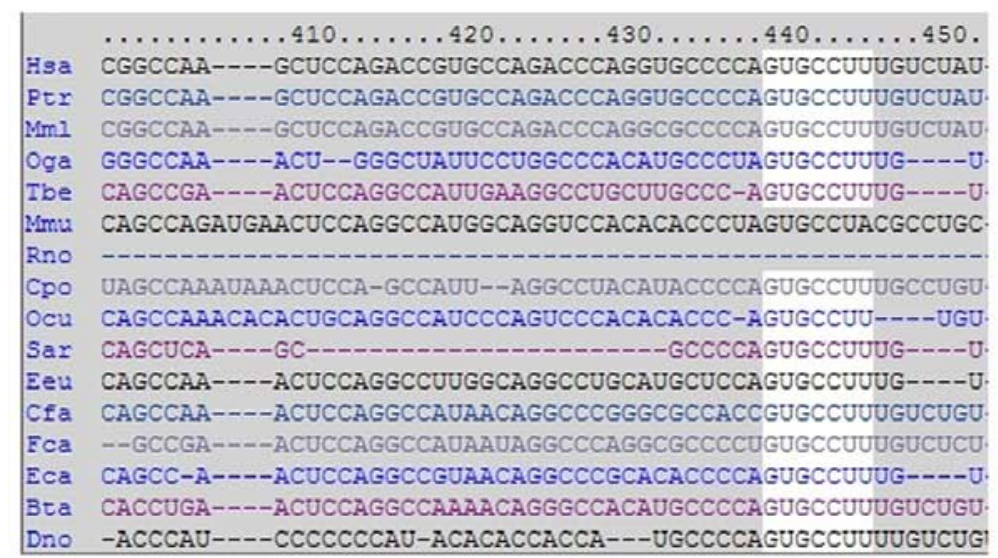

D
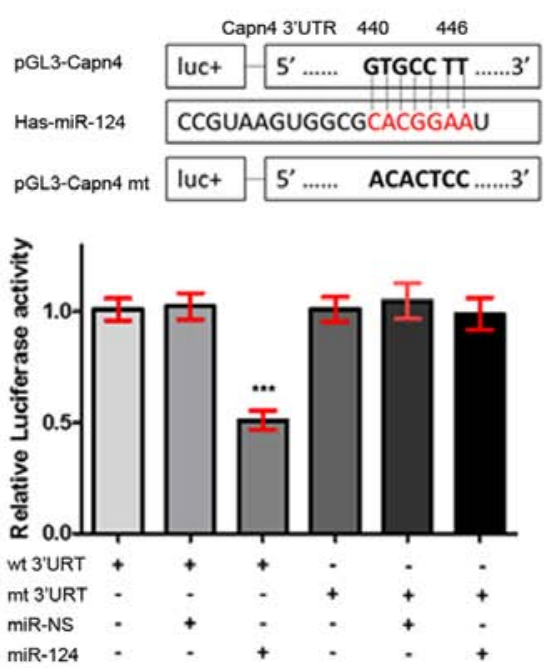

Figure 2. miR-124 directly targets the 3'-untranslated region (3'-UTR) of Capn4. (A) Candidate target genes of miR-124 are predicted using TargetScan and the human microRNA database. The nucleotide 440-446 region within the 3'-UTR of the Capn4 gene contains the candidate binding site for miR-124. (B) BibiServ analysis of free energy (DG) for hybridization of the Capn4 3'-UTR and miR-124. (C) Homology analysis of the nucleotide 440-446 region within the 3'-UTR of the Capn4 gene across species. (D) HEK293T cells were co-transfected with pGL3-Capn4-wt and miR-124 mimics, pGL3-Capn4-mt and miR-124 mimics or pGL3-Capn4-wt and scrambled miRNA controls (NS). Luciferase activities were then determined. Data are shown as mean \pm SD of at least three independent experiments in triplicate. ${ }^{* * *} \mathrm{P}<0.001$.

A
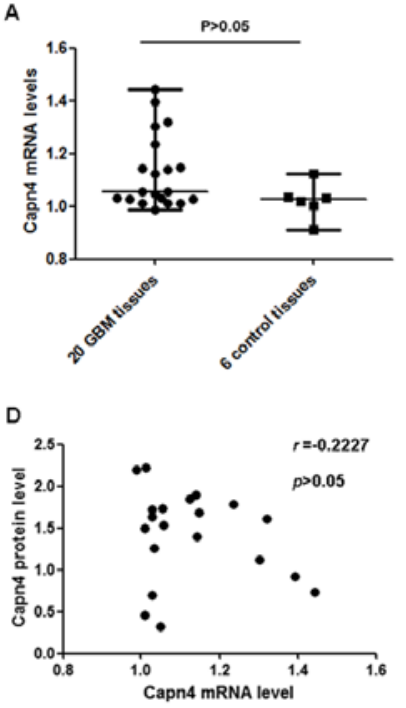

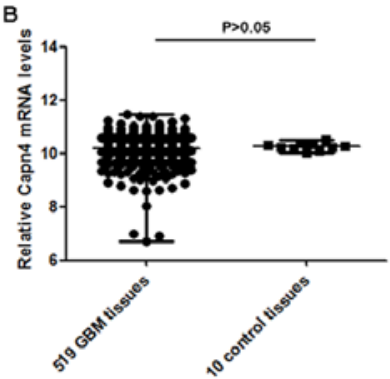

E

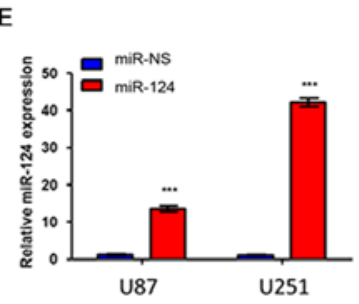

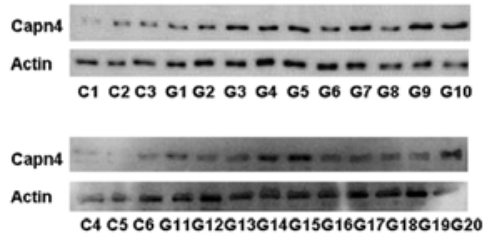

C4 C5 C6 G11G12G13G14G15G16G17G18G19G20

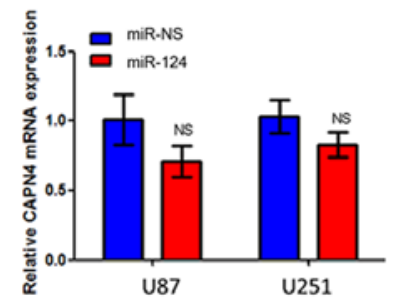

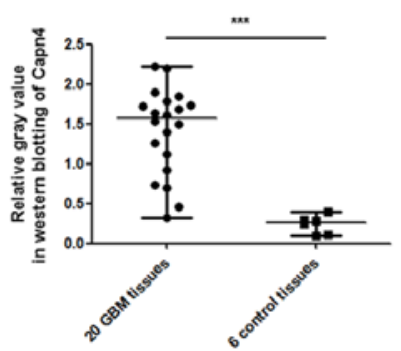

G

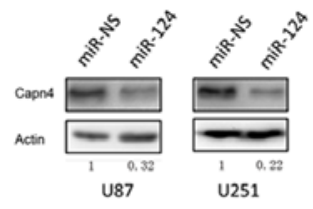

Figure 3. Capn4 is upregulated in GBM tissues or cells at the posttranscriptional level. (A) RT-qPCR assays were performed to determine the mRNA transcript levels of Capn4 in 20 GBM tissue and 6 normal brain tissue specimens. (B) Data from the TCGA was queried, yielding 519 GBM tissue and 10 normal unmatched brain tissue specimens. (C) Capn 4 expression in 20 GBM tissue and 6 normal brain tissue specimens was examined by immunoblotting assays. Capn 4 expression was normalized against $\beta$-actin. $C_{1}-C_{6}$, normal controls; $G_{1}-G_{20}$, GBM tissues. (D) Spearman's correlation analysis of mRNA transcript levels and protein levels of Capn4 in GBM tissues. (E and F) U87 and U251 cells were transfected with miR-124 mimics or scrambled miRNA (miR-NS) and miR-124 (E) and Capn4 mRNA transcript levels (F) were determined by RT-qPCR assays. (G) Capn4 expression in transfected U87 and $\mathrm{U} 251$ cells was examined by immunoblotting assays. Data in (A-C) are expressed as median and range. Data in (E) and (F) are shown as mean \pm SD of at least three independent experiments in triplicate. $\mathrm{P}>0.05, \mathrm{GBM}$ vs. controls in (A) and (B) and ${ }^{* * * *} \mathrm{P}<0.001, \mathrm{GBM}$ vs. controls in $(\mathrm{C})$ and $(\mathrm{E})$. 
A

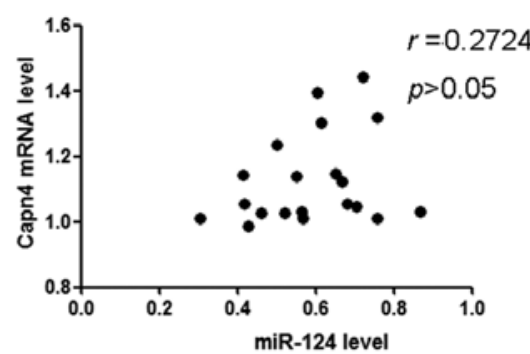

B

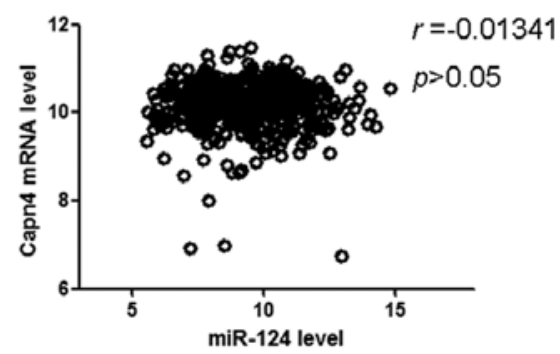

C

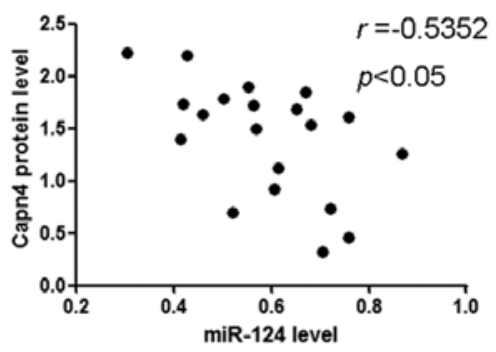

Figure 4. miR-124 expression correlates negatively with Capn4 protein levels in GBM. Spearman's correlation analysis of Capn4 mRNA transcript levels and miR-124 levels in (A) GBM tissues from our cohort and (B) the queried data from the TCGA and (C) of Capn4 protein level and miR-124 levels in GBM tissues from our cohort.

A
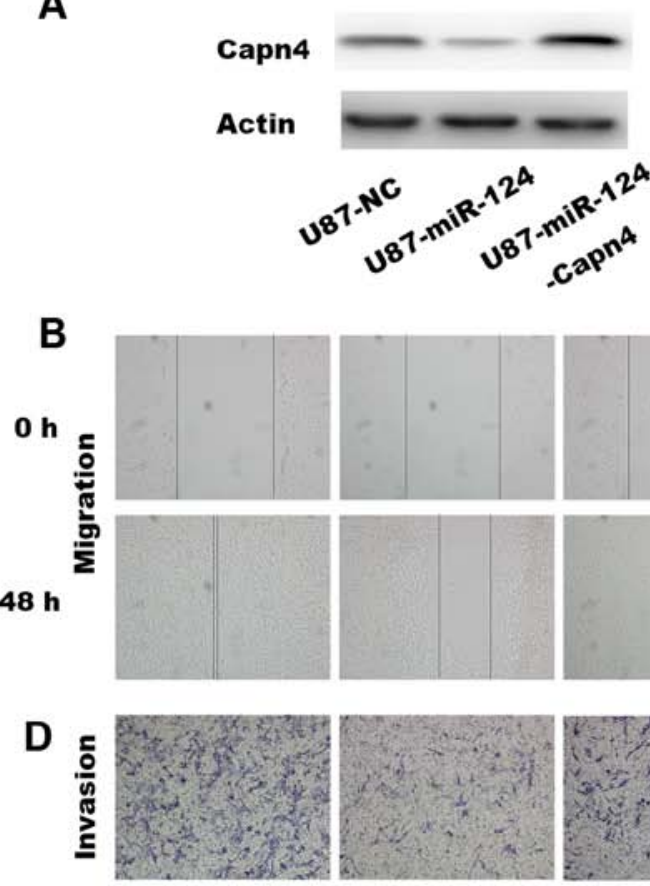

U87-NC
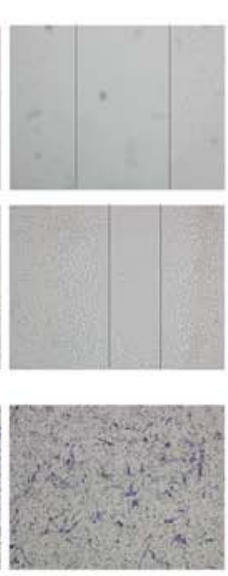

U87-miR-124
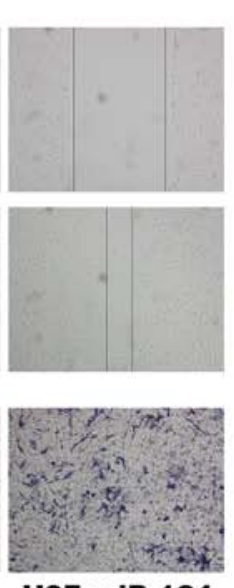

U87-miR-124

-Capn4
$\mathbf{F}$

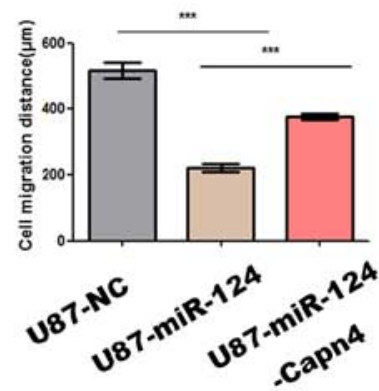

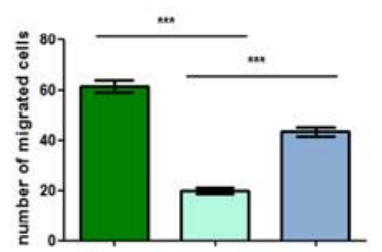

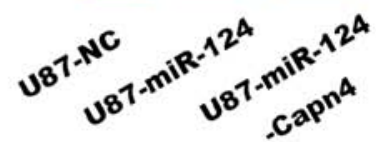

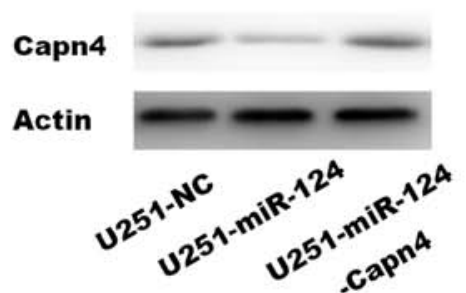

C h

$48 h$

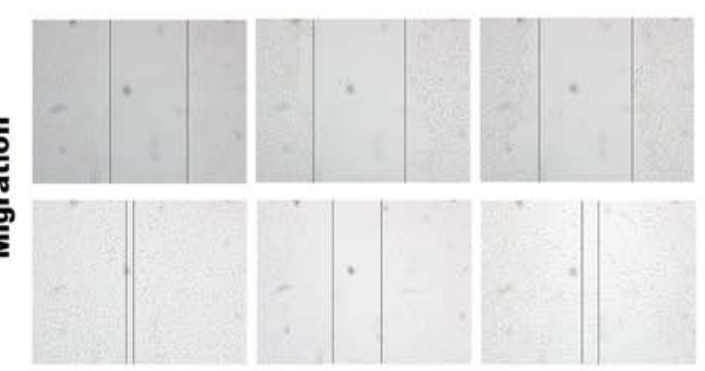

E
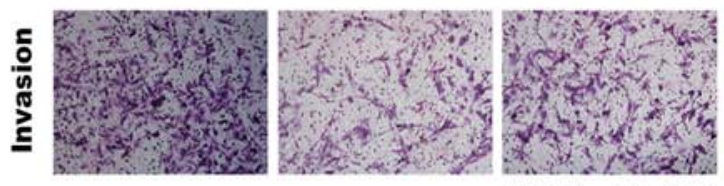

U251-NC

U251-miR-124

U251-miR-124

-Capn 4
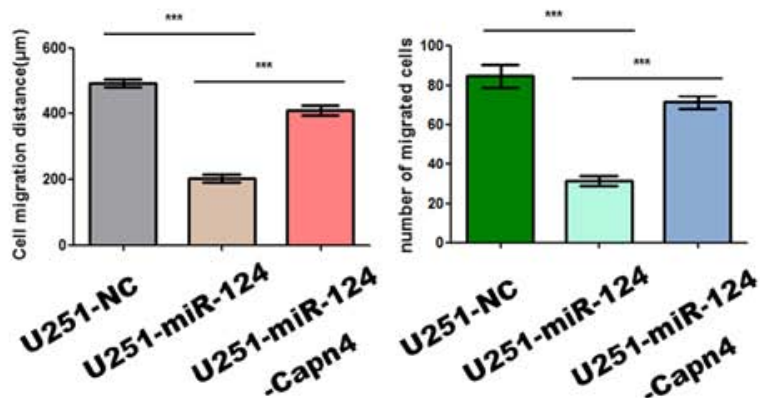

Figure 5. miR-124 suppresses the migration and invasion of glioma cells by modulating Capn4. U87 and U251 cells were transfected with scrambled microRNA (NC), miR-124 mimics, or miR-124 mimics together with pLenti-Capn4 as described in Materials and methods. (A) The Capn4 protein level in transfected U87 (left panel) and U251 cells (right panel) was examined by immunoblotting. Representative images are shown of at least three independent experiments. Cell motility was evaluated by wound-healing assays as described in Materials and methods. Representative images are shown of at least three independent experiments for (B) U87 and (C) U251 cells. Cell migration was evaluated by Transwell assays as described in Materials and methods. Representative images are shown of at least three independent experiments for U87 (D) and U251 cells (E). (F) Bar graphs are the mean \pm SD of three independent observations for U87 and U251 cells. ${ }^{* * *} \mathrm{P}<0.001$.

We determined whether Capn4 played any role in the miR-124-mediated suppression of the migration and inva- sion of glioma cells. Transfection of U87 and U251 cells with miR-124 mimics caused an apparent reduction in the level 
U87

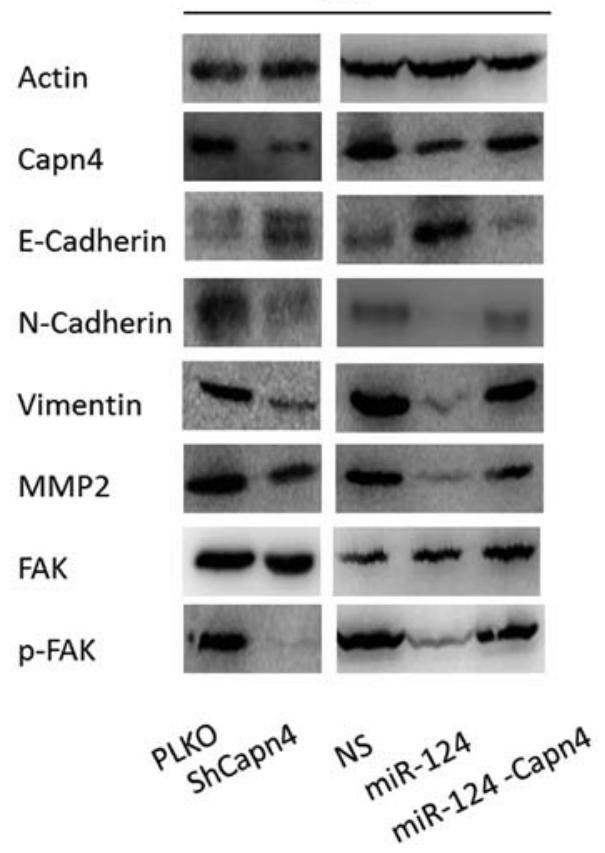

U251

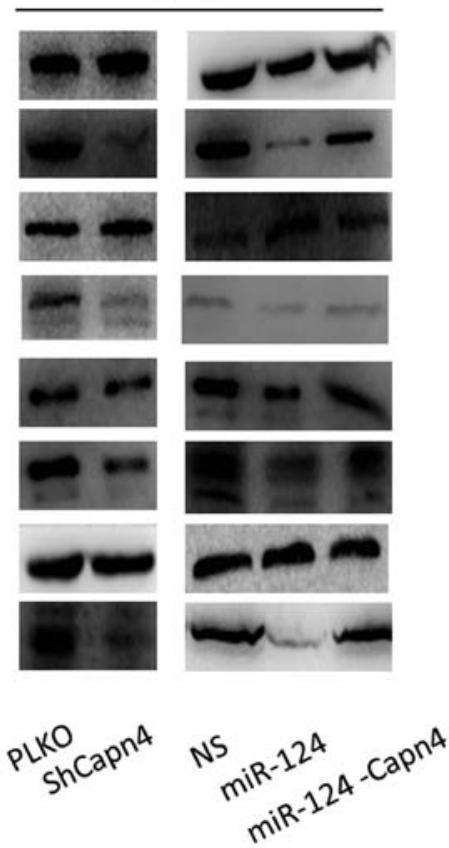

Figure 6. Capn4 is involved in the epithelial-mesenchymal transition (EMT) of glioma cells. U87 cells and U251 were transfected with plasmid pLKO or pLKO-shRNA-Capn4 and protein expression was examined by immunoblotting. U87 and U251 cells were transfected as in Fig. 5 and proteins involved in EMT were examined by immunoblotting. Representative images are shown of at least three independent experiments.

of Capn4 protein in the two cells, which, however, was abrogated by co-transfection with pLenti-Capn4 (Fig. 5A). The wound-healing assays showed that Capn4 downregulation by miR-124 mimics suppressed the migration of U87 (Fig. 5B) and $\mathrm{U} 251$ cells ( $\mathrm{P}<0.001$ vs. controls) (Fig. 5C). The decrease in the migration of U87 and U251 cells was markedly attenuated by co-transfection with pLenti-Capn4 ( $\mathrm{P}<0.001$ vs. miR-124 mimics) (Fig. 5F). In addition, Transwell assays demonstrated that miR-124 mimics significantly reduced the number of migrated U87 (Fig. 5D) and U251 cells ( $\mathrm{P}<0.001$ vs. controls) (Fig. 5E). The reduction in the number of migrated glioma cells was lessened by co-transfection with pLenti-Capn4 $(\mathrm{P}<0.001$ vs. miR-124 mimics) (Fig. 5F). The results suggested that miR-124 modulated the migration and invasion ability of gliomas cells via Capn4.

Capn4 is involved in the EMT of glioma cells. miR-124 has been demonstrated to reverse the EMT (16). We investigated whether Capn 4 was involved in this process. Capn 4 knockdown by shRNA was associated with an increase in E-cadherin and a decrease in $N$-cadherin and vimentin. Capn 4 downregulation also caused an apparent reduction in the level of phospho-FAK and MMP2 in U87 cells and U251 cells. Similar findings were observed in U87 cells and U251 cells transfected with miR-124 mimics. These changes were attenuated by co-transfection with pLenti-Capn4 (Fig. 6). The results indicated that Capn4 was involved in the EMT of glioma cells and was modulated by $\mathrm{miR}-124$ in the process.

\section{Discussion}

The highly infiltrative and invasive nature of malignant gliomasis a significant contributor to the poor outcome of the disease. miR-124 and Capn4 are involved in the migration and invasion of glioma cells and their aberrant expression in glioma tissues has been found to be associated with the outcome of the disease $(7,10)$. To the best of our knowledge, the present study provides the first piece of evidence that miR-124 directly targets Capn4 in modulating the migratory and invasive activities of glioma cells in vitro. Additionally, miR-124 and Capn 4 are aberrantly expressed in GBM tissues and miR-124 expression negatively correlates with Capn4 protein levels. Understanding the molecular mechanisms of gliomagenesis and tumor progression is necessary to explore more effective diagnostic and therapeutic strategies. In a previous study, it was shown that alterations in Capn4 expression correlated with glioma grade and patient survival (13). In the present study, we have shown that the Capn4 protein levels, but not the mRNA levels, in GBM were higher than the control tissues, which is consistent with our immunohistochemical findings in our previous report (13).

Currently, studies on the role of miR-124 in glioma involve only a small number of patients. Zhao et al studied 9 GBM patients (6) while An et al studied 16 glioma patients (10) including eight high-grade glioma patients. We studied 20 archived GBM tissue specimens and showed that miR-124 was significantly downregulated while Capn4 was markedly upregulated in GBM tissues. This finding was further supported by our analysis of 519 GBM patients in TGCA. We and other authors have shown that miR-124 suppresses the migration and invasion of glioma cells. An et al found that this inhibitory effect of miR-124 on glioma cell migration and invasion was exerted via ROC1 (10), a protein involved in the regulation of cell motility. By contrast, Zhao et al, showed that miR-124 inhibited the invasiveness of glioma cells by targeting PPP1R3L (6), an inhibitory member of the apoptosis-stimulating protein 
of the p53 family that also regulates growth, cell cycle progression, metastasis and apoptosis of various types of cancer. The role of Capn 4 in regulating the invasiveness of glioma cells has not been well elucidated, as identified in our previous study, where the results demonstrated that downregulation of Capn4 suppressed the invasion and migration of glioma cells in vitro. To the best of our knowledge, no other study has focused on the role of Capn 4 in glioma. In this study, we demonstrated that miR-124 targeted Capn4 in regulating the migration and invasion of glioma cells.

Dai et al, demonstrated that Capn4 promoted the metastasis of hepatocellular carcinoma cells via activation of the FAK-Src signaling pathway and MMP2 (17). We also found that Capn 4 downregulation by shRNA was associated with reduced levels of phospho-FAK and MMP2. Similarly, miR-124 mimics suppressed the levels of phospho-FAK and MMP2 in U87 cells, which was attenuated by co-transfection with plasmid vectors expressing Capn4. An increasing number of studies have shed light on the mechanisms whereby glioma cells infiltrate normal brain. Glioma invasion is a multistep process that involves detachment from the tumor mass, remodeling of the extracellular matrix, and migration of glioma cells (18). Several factors, including CD44 (19), cadherins (20), and matrix metalloproteinases (21), have been shown to mediate these processes. In this study, the results showed that the exogenous overexpression of miR-124 suppressed the mesenchymal phenotype of U87 cells by inhibiting Capn 4 .

In conclusion, this study has demonstrated the functions of miR-124 in inhibiting the invasive propensity of glioma cells. miR-124 controls the invasive phenotype of glioma cells via one of its downstream targets, Capn4. In view of its frequent downregulation in GBM, it is likely that brain-enriched miR-124 may not only be important for neural development but may also serve as a putative tumor suppressor miRNA that prevents gliomagenesis by inhibiting the invasiveness of glioma cells. The results suggest a new avenue for elucidating the molecular mechanism of glioma cell invasion and developing therapeutic strategies against glioma by restoring the level of miR-124 and suppressing Capn4.

\section{Acknowledgements}

This study was supported by 'China National Funds for Distinguished Young Scientists' (81025013 to Ying Mao), 'The major research and development project of innovative drugs, Ministry of Science and Technology' (2012ZX09303004-001) and Shanghai Municipal Commission of Health and Family Planning (20124354).

\section{References}

1. Stupp R, Mason WP, van den Bent MJ, Weller M, Fisher B, Taphoorn MJ, Belanger K, Brandes AA, Marosi C, Bogdahn U, et al; European Organisation for Research and Treatment of Cancer Brain Tumor and Radiotherapy Groups; National Cancer Institute of Canada Clinical Trials Group: Radiotherapy plus concomitant and adjuvant temozolomide for glioblastoma. N Engl J Med 352: 987-996, 2005.
2. Van Meir EG, Hadjipanayis CG, Norden AD, Shu HK, Wen PY and Olson JJ: Exciting new advances in neuro-oncology: The avenue to a cure for malignant glioma. CA Cancer J Clin 60: 166-193, 2010.

3. Riddick $\mathrm{G}$ and Fine HA: Integration and analysis of genomescale data from gliomas. Nat Rev Neurol 7: 439-450, 2011.

4. Wang Z, Wang B, Shi Y, Xu C, Xiao HL, Ma LN, Xu SL, Yang L, Wang QL, Dang WQ, et al: Oncogenic miR-20a and miR-106a enhance the invasiveness of human glioma stem cells by directly targeting TIMP-2. Oncogene 34: 1407-1419, 2015.

5. Mao J, Zhang M, Zhong M, Zhang Y and Lv K: MicroRNA-204, a direct negative regulator of ezrin gene expression, inhibits glioma cell migration and invasion. Mol Cell Biochem 396: 117-128, 2014.

6. Zhao WH, Wu SQ and Zhang YD: Downregulation of miR-124 promotes the growth and invasiveness of glioblastoma cells involving upregulation of PPP1R13L. Int J Mol Med 32: 101-107, 2013.

7. Xia H, Cheung WK, Ng SS, Jiang X, Jiang S, Sze J, Leung GK, Lu G, Chan DT, Bian XW, et al: Loss of brain-enriched miR-124 microRNA enhances stem-like traits and invasiveness of glioma cells. J Biol Chem 287: 9962-9971, 2012.

8. Li D, Chen P, Li XY, Zhang LY, Xiong W, Zhou M, Xiao L, Zeng F, Li XL, Wu MH, et al: Grade-specific expression profiles of miRNAs/mRNAs and docking study in human grade I-III astrocytomas. OMICS 15: 673-682, 2011.

9. Sonntag KC, Woo TU and Krichevsky AM: Converging miRNA functions in diverse brain disorders: A case for miR-124 and miR-126. Exp Neurol 235: 427-435, 2012.

10. An L1, Liu Y, Wu A and Guan Y: microRNA-124 inhibits migration and invasion by down-regulating ROCK1 in glioma. PLoS One 8: e69478, 2013.

11. Storr SJ, Carragher NO, Frame MC, Parr T and Martin SG: The calpain system and cancer. Nat Rev Cancer 11: 364-374, 2011.

12. Zhang C, Bai DS, Huang XY, Shi GM, Ke AW, Yang LX, Yang XR, Zhou J and Fan J: Prognostic significance of Capn4 overexpression in intrahepatic cholangiocarcinoma. PLoS One 8: e54619, 2013

13. Zhuang Q, Qian X, Cao Y, Fan M, Xu X and He X: Capn4 mRNA level is correlated with tumour progression and clinical outcome in clear cell renal cell carcinoma. J Int Med Res 42: 282-291, 2014.

14. Cai JJ, Qi ZX, Hua W, Zhu JJ, Zhang X, Yao Y and Mao Y: Increased expression of Capn 4 is associated with the malignancy of human glioma. CNS Neurosci Ther 20: 521-527, 2014.

15. Bai DS, Dai Z, Zhou J, Liu YK, Qiu SJ, Tan CJ, Shi YH, Huang C, Wang Z, He YF, et al: Capn4 overexpression underlies tumor invasion and metastasis after liver transplantation for hepatocellular carcinoma. Hepatology 49: 460-470, 2009.

16. Qin W, Pan Y, Zheng X, Li D, Bu J, Xu C, Tang J, Cui R, Lin P and $\mathrm{Yu}$ X: MicroRNA-124 regulates TGF- $\alpha$-induced epithelialmesenchymal transition in human prostate cancer cells. Int $\mathrm{J}$ Oncol 45: 1225-1231, 2014.

17. Dai Z, Zhou SL, Zhou ZJ, Bai DS, Xu XY, Fu XT, Chen Q, Zhao YM, Zhu K, Yu L, et al: Capn4 contributes to tumour growth and metastasis of hepatocellular carcinoma by activation of the FAK-Src signalling pathways. J Pathol 234: 316-328, 2014.

18. Cuddapah VA, Robel S, Watkins S and Sontheimer H: A neurocentric perspective on glioma invasion. Nat Rev Neurosci 15: 455-465, 2014.

19. Pietras A, Katz AM, Ekström EJ, Wee B, Halliday JJ, Pitter KL, Werbeck JL, Amankulor NM, Huse JT and Holland EC: Osteopontin-CD44 signaling in the glioma perivascular niche enhances cancer stem cell phenotypes and promotes aggressive tumor growth. Cell Stem Cell 14: 357-369, 2014.

20. Barami K, Lewis-Tuffin L and Anastasiadis PZ: The role of cadherins and catenins in gliomagenesis. Neurosurg Focus 21: E13, 2006.

21. Könnecke $H$ and Bechmann I: The role of microglia and matrix metalloproteinases involvement in neuroinflammation and gliomas. Clin Dev Immunol 2013: 914104, 2013. 